\title{
Stereotactic Radiofrequency Ablation of Trigeminal Ganglion with Intraoperative CT Scans and under General Anesthesia
}

\author{
Arun-Angelo Patil1 ${ }^{*}$, Andrea Jennifer Chamczuk ${ }^{1}$, Benjamin Nelson ${ }^{2}$ \\ ${ }^{1}$ Creighton University Medical Center, Omaha, NE, USA \\ ${ }^{2}$ Creighton University School of Medicine, Omaha, NE, USA \\ Email: *arun.patil@alegent.org
}

Received 10 December 2015; accepted 22 January 2016; published 25 January 2016

Copyright (C) 2016 by authors and Scientific Research Publishing Inc.

This work is licensed under the Creative Commons Attribution International License (CC BY). http://creativecommons.org/licenses/by/4.0/

(c) (7) Open Access

\section{Abstract}

Introduction: Traditionally radiofrequency ablation of the trigeminal ganglion is performed using fluoroscopy and under heavy sedation. Intra-operative stimulation studies are used in order to confirm the presence of the probe tip in the appropriate division. Unfortunately, in older patients it becomes challenging to transition between heavy sedation (for probe insertion and lesion generation) and light sedation (for stimulation studies). To solve this problem, the authors describe a method in which the procedure was performed under general anesthesia and appropriate needle position was confirmed by intraoperative CT scans. Furthermore, to make the procedure more accurate a stereotactic frame was used. Methods: Eleven procedures were performed on 10 patients from 2012-2015 with a median follow-up of 21 months. The age range of the patients was 33 - 90 years (median of 55), and with a male to female ratio of 6:4. Ablations were accomplished on the 3rd division in five patients, the 2 nd division in three patients, and 2 nd and 3rd divisions in two patients (one of them underwent bilateral procedures). The procedures were performed under general anesthesia, using a Patil stereotactic frame on the CT table. The center of the foramen ovale was chosen as the target. The probe insertion point was approximately $2.5 \mathrm{~cm}$ lateral to the angle of the mouth. To place the probe tip in the 3rd division it was advanced through the foramen ovale into the trigeminal ganglion until its tip was $5 \mathrm{~mm}$ anterior to the clival plane. To place it in the 2nd division, it was advanced until it was in the clival plane. The probe had an exposed tip of 8 $\mathrm{mm}$ and heating was done at $75^{\circ} \mathrm{C}$ for 60 seconds. Results: Nine patients are pain-free; one patient, who had atypical facial pain did not benefit from the procedure; and one patient (with bilateral pain) has hyperesthesia on one side. Conclusion: Trigeminal ganglion ablation under general anesthesia is a good option for older patients and for those who cannot tolerate being awakened during the procedure. The stereotactic method enables easy placement of the probe and intraoperative CT scans affords confirmation of accurate probe placement.

\footnotetext{
${ }^{*}$ Corresponding author.
} 
Keywords

Trigeminal Neuralgia, Stereotactic, Intraoperative Scans, General Anesthesia, Radiofrequency Ablation

\section{Introduction}

Trigeminal neuralgia (TN) is one of the most exquisitely painful syndromes known to humankind. Patients suffering from TN describe pain episodes as "sharp, shooting, stabbing, or lightning-bolt" sensations along the distribution of cranial nerve $\mathrm{V}$ that last from a few seconds up to two minutes. Most individuals suffer from unilateral disease, but bilateral pain can also occur. The etiology of this pain includes vascular compression of the nerve as it exits the brain stem, mass effect from a tumor, trauma to the nerve, infection, amyloidosis, infarction in the brainstem and demyelination of the nerve root due to multiple sclerosis (MS). While familial cases have been described, the vast majority are idiopathic. Imaging studies may demonstrate tumor, MS plaque, vascular loop around the nerve; or the studies may be completely normal.

Approximately one out of four patients fails medical treatment [1]. For these medically refractory patients, surgical intervention is indicated. The first surgical procedure used to treat tic douloureux was performed in the 1850s by Carnochan, which included a disfiguring transantral approach to the gasserian ganglion through the maxillary sinus [2]. Years later, Hartley and Krause developed a strategy to enter from within the cranium. Cushing developed an operation that better identified the ganglion and reduced mortality from exsanguination by reflecting the middle meningeal artery away from the site of operation. Dandy entered through the cerebellum, and provided the first evidence of cases where vascular compression of the nerve root was responsible for the painful symptoms [2]. Sweet was the first to use thermocoagulation to destroy pain fibers in TN [3]. Today, targeted radiation (gamma-knife), open endoscopic, and several variations of percutaneous surgery are performed. Open surgery is directed at relieving vascular compression of the nerve, whereas gamma-knife and percutaneous surgery are aimed at nerve destruction. The percutaneous approaches to obliterate the irritated trigeminal nerve are subdivided into balloon compression, glycerol injection, and electrocoagulation (radiofrequency ablation) procedures. Of these percutaneous options, radiofrequency ablation is the most commonly utilized, especially in elderly patients [4].

The traditional percutaneous radiofrequency ablation approach places the patient under light sedation and uses fluoroscopy with intraoperative X-rays to confirm the presence of the probe tip within the gasserian ganglion [5]. This procedure requires patients to be awake in order to respond to nerve stimulation prior to ablation, which is an uncomfortable proposition for some patients. Furthermore, intraoperative stimulation tests rely on the patient's verbal response under a state of partial sedation, complicating the accuracy of this subjective response. To obviate this difficulty, some surgeons have performed the procedure under general anesthesia using fluoroscopic imaging [5]. Another paper described the use of anhidrotic response to determine the location of the probe tip under general anesthesia [4].

Here, we describe a procedure, under general anesthesia, in which a stereotactic frame was used to place the probe at the target and intraoperative CT images were used to confirm accurate probe placement.

\section{Materials and Methods}

Eleven procedures were performed on 10 patients from 2012-2015 with a median follow-up of 21 months. The age range of the patients was 33 - 90 years (median of 55), and with male to female ratio of 6:4. Ablations were accomplished on the 3rd division in five patients, the 2nd division in three patients, and 2nd and 3rd divisions in two patients (one of them underwent bilateral procedures). The procedures were performed under general anesthesia, using a Patil stereotactic frame on the CT table. The center of the foramen ovale was chosen as the target. The probe insertion point was approximately $2.5 \mathrm{~cm}$ lateral to the angle of the mouth. To place the probe tip in the 3rd division it was advanced through the foramen ovale (Figure 1) into the trigeminal ganglion until its tip was $5 \mathrm{~mm}$ anterior to the clival plane (Figure 2). To place it in the 2nd division, it was advanced until it was in the clival plane (Figure 3). The probe was a 20 gauge cannula with an exposed tip of $10 \mathrm{~mm}$ (manufactured by Neuro Therm, Wilmington, MA). The probe tip was heated at $75^{\circ} \mathrm{C}$ for 60 seconds. 
Results: The results are summarized in Table 1. Nine patients (90\%) are pain-free on follow-up; one patient (10\%), who had atypical facial pain did not benefit from the procedure; and one patient (with bilateral pain) has hyperesthesia unilaterally. None of the patients reported post-operative numbness in the unintended divisions.

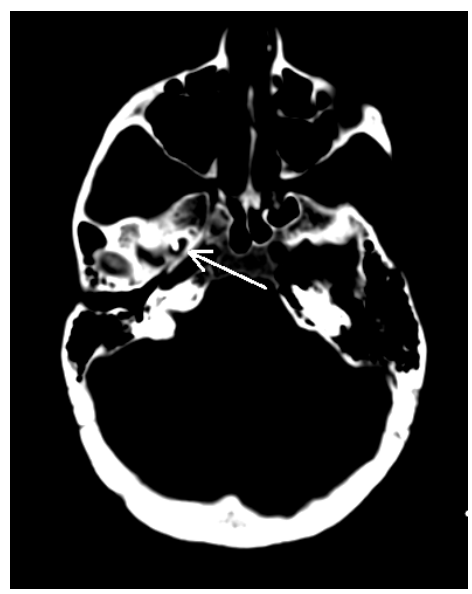

Figure 1. The arrow points to the foramen ovale on the right side. The probe tip is visible in it.

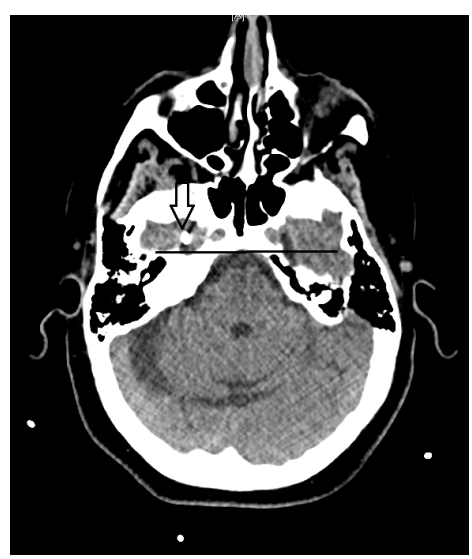

Figure 2. The horizontal line represent the clival plane. The probe tip is $5 \mathrm{~mm}$ anterior to it (arrow).

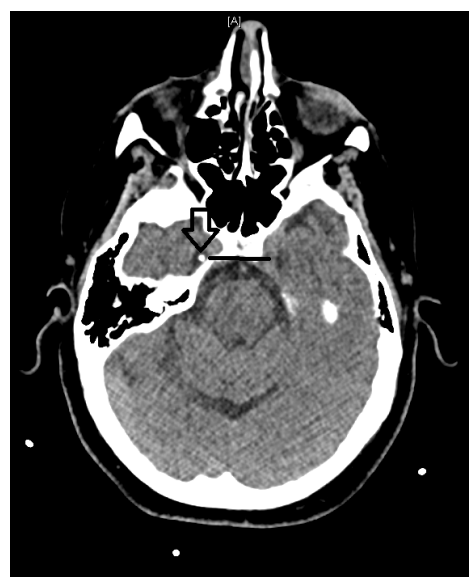

Figure 3. The horizontal line marks the clival plane. The probe tip at this plane (arrow). 
Table 1. Patient characteristics and case series results of intraoperative CT-guided stereotactic radio ablation under general anesthesia.

\begin{tabular}{cccll}
\hline Patient & Age & Sex & Trigeminal nerve involvement & Post-op pain \\
\hline 1 & 32 & F & V2 and V3 & Pain-free \\
2 & 87 & M & V2 and V3 \\
(repeat procedure after 1.5 years of relief) & Pain-free \\
3 & 90 & F & V2 & Pain-free \\
4 & 33 & M & V2 (atypical facial pain) & No relief \\
5 & 56 & M & V3 & Pain-free \\
6 & 55 & F & V3 (bilateral) & Pain-free right side; \\
& & & & hyperesthesia left side \\
7 & 70 & M & V3 (post gamma-knife radiation without benefit) & Pain-free \\
8 & 35 & M & V2 (traumatic pain) & Pain-free \\
9 & 78 & M & V3 & Pain-free \\
10 & 53 & F & V3 (multiple sclerosis) & Pain-free \\
\hline
\end{tabular}

\section{Discussion}

Microvascular decompression (MVD), "gamma-knife” radiation (GKR), and percutaneous nerve destruction are the three main types of procedural intervention used to treat trigeminal neuralgia (TN) [6]. Among the different methods to treat TN, percutaneous radiofrequency ablation (RFA) has the highest rates of complete pain relief especially in elderly patients [6]-[8]. It has been demonstrated that RFA provides immediate relief for patients, has lower rates of complications, and has less mortality in elderly patients compared to MVD and GKR [6]. As the majority of TN patients are elderly, optimization of the RFA procedure is of utmost importance.

The traditional percutaneous approach to RFA included intraoperative fluoroscopy with X-ray to locate the Gasserian ganglion [2] [4] [5] [8]. The relationship of the probe-tip to individual trigeminal division is based on the position of the probe-tip to the junction of the petrous ridge and clivus. If the probe-tip $5 \mathrm{~mm}$ anterior to this junction, then it is in the third division. If the probe-tip is at the junction of the petrous ridge and clivus, then it is in the second division. Furthermore, stimulation studies are performed to confirm the location of the probe-tip. This method requires partial sedation in order to perform intraoperative stimulation tests prior to nerve destruction [8] [9]. Managing patient sedation to an adequate level to achieve a reliable verbal response to nerve stimulation is notoriously problematic, especially in elderly patients whose hypersensitivity to sedatives results in a very narrow window in which light sedation can be adequately maintained [10].

There are several approaches described in the literature to perform the procedure completely under general anesthesia. Hart et al. performed the whole procedure with the patient asleep and without the stimulation studies [5]. They determined the position of the probe-tip in the trigeminal ganglion purely based on X-ray images. In their study of 44 patients with a follow-up of $2-3$ years they found comparable pain relief compared to literature review of traditional RFA surgery with stimulation testing and light sedation [5]. Karol and colleagues used sensory and motor trigeminal evoked potentials to localize trigeminal electrodes during the RFA procedure, under general anesthesia [12]. Another method to determine the position of the probe-tip in the trigeminal ganglion under general anesthesia was to use antidromic response [4].

In our previous publication, we reported use of stereotactic frame to place the probe-tip at the target, intraoperative CT scan to confirm accurate placement of the probe-tip at the target, and stimulation studies to re-confirm it [11]. Using that protocol however, we encountered difficulties in titrating sedatives to maintain proper sedation levels in older patients. Therefore, we began using general anesthesia for the entire procedure and abandoned using stimulation studies. None of the patients reported numbness in the unintended divisions and $80 \%$ reported freedom from pain.

The advantages of the method described in this paper are several. The patient is completely under general anesthesia. This avoids painful moments to the patient during the procedure. Furthermore, since the patient is intubated, the risk of too much sedation is avoided. Use of intraoperative CT enables the surgeons to visualize the foramen ovale with ease. The use of stereotactic approach makes it easy to insert the probe into the foramen 
ovale. The main disadvantage is, use of CT room time; which may be hard to find in some hospitals.

\section{Conclusion}

The authors report a small series in which radiofrequency ablation of trigeminal ganglion was successfully performed under general anesthesia using a stereotactic frame, and anatomical location of the probe-tip with intraoperative CT scans was the only guide to ascertain accurate probe placement. The results are comparable to those obtained where stimulation studies were employed. These results therefore challenge the necessity of painful intraoperative stimulation tests for RFA in TN.

\section{Ethics Committee Approval}

The author has performed this procedure over the past 10 years. The only modification in this paper was the use of general anesthesia. Ethics committee approval was, therefore not needed.

\section{References}

[1] Holland, M., Noeller, J., Buatti, J., He, W., Shivapour, E.T., et al. (2015) The Cost-Effectiveness of Surgery for Trigeminal Neuralgia in Surgically Naïve Patients: A Retrospective Study. Clinical Neurology and Neurosurgery, 137, 34-37. http://dx.doi.org/10.1016/j.clineuro.2015.06.011

[2] Kellogg, R., Pendelton, C., Quinones-Hinojosa, A. and Cohen-Gadol, A. (2010) Surgical Treatment of Trigeminal Neuralgia: A History of Early Strides toward Curing a "Cancerous Acrimony”. Neurosurgery, 67, 1419-1425. http://dx.doi.org/10.1227/NEU.0b013e3181f0ef13

[3] Sweet, W.H. and Wepsic, J.G. (1974) Controlled Thermocoagulation of Trigeminal Ganglion and Rootlets for Differential Destruction of Pain Fibres, Part I, Trigeminal Neuralgia. Journal of Neurosurgery, 40, 143-156.

[4] Bendersky, M., Hem, S., Landriel, F., Muntadas, J., Kitroser, M., et al. (2012) Identifying the Trigeminal Nerve Branches for Transovale Radiofrequency Thermolesion: “No Pain, No Stress”. Neurosurgery, 70, 259-263.

[5] Hart, M.G., Nowell, M. and Coakham, H.B. (2012) Radiofrequency Thermocoagulation for Trigeminal Neuralgia without Intra-Operative Patient Waking. British Journal of Neurosurgery, 26, 392-396. http://dx.doi.org/10.3109/02688697.2012.673650

[6] Lopez, B.C., Hamlyn, P.J. and Zakrzewska, J.M. (2004) Systematic Review of Ablative Neurosurgical Techniques for the Treatment of Trigeminal Neuralgia. Neurosurgery, 54, 973-982; discussion 982-983. http://dx.doi.org/10.1227/01.NEU.0000114867.98896.F0

[7] Oomens, M.A. and Forouzanfar, T. (2015) Pharmaceutical Management of Trigeminal Neuralgia in the Elderly. Drugs \& Aging, 32, 717-726. http://dx.doi.org/10.1007/s40266-015-0293-6

[8] Emril, D.R. and Kok-Yuen, H. (2010) Treatment of Trigeminal Neuralgia: Role of Radiofrequency Ablation. Journal of Pain Research, 3, 249-254.

[9] Kanpolat, Y., Deda, H., Akyar, S., Caglar, S. and Bilgic, S. (1989) CT-Guided Trigeminal Tractotomy. Acta Neurochirurgica, 100, 112-114. http://dx.doi.org/10.1007/BF01403596

[10] Thompson, E.M., Burchiel, K.J. and Raslan, A.M. (2013) Percutaneous Trigeminal Tractotomy-Nucleotomy with Use of Intraoperative Computed Tomography and General Anesthesia: Report of 2 Cases. Neurosurgical Focus, 35, E5. http://dx.doi.org/10.3171/2013.6.FOCUS13218

[11] Patil, A.A. (2010) Stereotactic Approach to the Trigeminal Ganglion Using a Stereotactic Frame and Intraoperative Computed Tomography Scans: Technical Note. Stereotactic and Functional Neurosurgery, 88, 277-280. http://dx.doi.org/10.1159/000316759

[12] Karol, E.A., Sanz, O.P. and Rey, R.D. (1991) Sensory and Motor Trigeminal Evoked Potentials to Localize the Position of Trigeminal Electrodes. Acta Neurochirurgica, 108, 110-115. http://dx.doi.org/10.1007/BF01418517

\section{Abbreviations}

CT: computed tomography; TN: trigeminal neuralgia; MVD: microvascular decompression; RFA: radiofrequency ablation; GKR: gamma knife radiation. 